\title{
Consensus of the Brazilian Society of Infectious Diseases on the Management and Treatment of Hepatitis C
}

Evaldo Stanislau Affonso de Araújo, João Silva Mendonça, Antonio Alci Barone, Fernando Lopes Gonçales Junior, Marcelo Simão Ferreira*, Roberto Focaccia, Jean-Michel Pawlotsky§* and Brazilian Society of Infectious Diseases HCV Consensus Group

Each year, and every day, the results of clinical trials and basic research provide us with a great deal of new information regarding viral hepatitis. We on the Viral Hepatitis Committee of the Sociedade Brasileira de Infectologia (SBI, Brazilian Society of Infectious Diseases) have been working to standardize the major issues surrounding dayto-day practice in treating patients infected with the hepatitis B or $\mathrm{C}$ virus (HBV or HCV). We have decided to address, in alternate years, HBV, together with hepatitis delta (HDV), and HCV, in our annual 'Consensus' on clinical management. Last year, we published the first HBV Consensus (BJID, 2007 (11):2-6). This year, we submit our HCV Consensus, which primarily serves to update the 2002 and 2004 SPI Consensuses. We distributed the principal topics among the Committee members, revised their work and compiled it into a Proceedings Supplement (to be published together with the BJID), which elucidates the highlights of the Consensus. A deeper review was written and referenced (it is our advice to the reader to read the Proceedings as well). A meeting was then held in Mogi das Cruzes in order to discuss, in a very practical and directed way, the issues most relevant to the Consensus, from public policies to the most complex therapeutic points. The results are summarized in a question/ answer, topic/statement format in this issue of the BJID. The main message of our statement was that we need to have the courage to act in favor of life. Many of us have adopted certain practices based on very new knowledge despite a lack of formal or official policies to support such practices. Some of us have been awaiting new compounds while patients are dying of chronic liver diseases. Unfortunately, the news from the battlefield is not so good. New compounds have been very disappointing (low potency, viral resistance, ineffective without interferon and various side effects, some serious). It is also difficult to incorporate new policies into everyday practice. However, strategies such as optimizing the use of pegylated interferon/ribavirin and encouraging treatment compliance, as well as finding new ways to monitor and slow liver disease progression, are effective and should be put into practice. Most importantly, the lowdose maintenance of pegylated interferon seems to be very promising, and the use of interferon alpha has saved lives. That is why we choose to expound upon what we believe to be the current standard of care and the gold standard for dealing with this hard to treat virus, as well as with the chronic complications of HCV infection. Our position will be reevaluated over the next two years. Until then, we are confident that our guide will be of great value to the readers. Finally, we would like to thank Roche and Schering Plough for the educational grants provided to the SBI. However, we must stress that neither company attempted to influence any of the decisions made by our consensus group.

The Brazilian Society of Infectious Diseases HCV Consensus Group

\section{Social and Collective Impact of the Decision-Making Process}

Serologic triage for hepatitis C virus (HCV): When and how? Diagnosis ‘campaigns'? Most vulnerable groups?

It is recommended that detection 'campaigns' be carried out for more vulnerable groups ${ }^{1}$, individuals in certain occupations (health professionals, technicians whose work potentially puts them in contact with blood), institutionalized individuals (prisoners, under-age felons, etc.), and the family members of patients with hepatitis C, as well as those infected with HIV. We emphasize that such individuals should be clearly informed that the triage diagnosis should be confirmed by a

\#Brazilian Society Infectious Diseases HCV Consensus Group: Ana Tereza Rodrigues Viso, Aline Vigani, André Cosme Oliveira, Carlos Eduardo de Melo, Carlos Brites Alves, Décio Diament, Edson Abdala, Edgard De Bortholi, Evandro Sobroza de Mello, Eduardo Sellan Gonçalves, Fátima Mitiko Tengan, Heloísa Pedrosa Mitre*, Kleber Dias Prado, Neiva Sellan Gonçales, Norma de Paula Cavalheiro, Rinaldo Focaccia Siciliano, Rodrigo Nogueira Angerami* , Umbeliana Barbosa de Oliveira, Venâncio Avancini Ferreira Alves*.

$\S$ Special guest.

* Members who did not participate of the Consensus Meeting occurred in the Blue Tree Park Hotel, Mogi das Cruzes/SP, on August 25 $5^{\text {th }}-26^{\text {th }}, 2007$. method with more sensitivity and specificity, as well as that confirmation of the diagnosis will not necessary imply treatment of the infection. Age is a factor to be considered, since older people are more likely to have been exposed to the disease over the course of their lifetime.

The infected health professional: are their activities restricted in any way?

A priori, the activity of health professionals with hepatitis $\mathrm{C}$ should not be restricted. However, strict application and educational measures are recommended regarding the biosafety guidelines, and it should be noted that more data are needed before a definitive recommendation can be made.

Is hepatitis $\mathrm{C}$ a sexually transmitted disease (STD)?

Although hepatitis C is not conceptually an STD, sexual transmission is possible, albeit uncommon, and the risk of

${ }^{1}$ History of blood product transfusion, history of major surgery, unsafe parenteral exposure (reusable syringes, sharing of utensils during the use of licit or illicit injected substances, undergoing diagnostic or esthetic therapeutic procedures involving reusable or inadequately sterilized material, e.g., tattoo, acupuncture, piercing, manicure, dental treatment, etc.), and sharing of utensils during the use of inhaled drugs. 
such transmission increases when the individual presents genital lesions or HIV positivity, as well as when the individual engages in risky sexual behavior. In such situations, the use of condoms is recommended. Heterosexual monogamous couples who present discrepant serologic results can use condoms of their own accord after being properly instructed. We would like to issue a warning regarding the risk of transmission in the household through the communal use of utensils contaminated with blood.

Hepatitis C during pregnancy and childbirth: To get pregnant or not? Cesarean or vaginal delivery? Breastfeeding?

Pregnancy is not contraindicated in women of childbearing age infected with $\mathrm{HCV}$, although contraindications related to the period of treatment should be respected. Regarding the type of delivery, the decision should be made by an obstetrician. It should be noted, however, that a high viral load of HCV can be a relevant factor in this decision. Nevertheless, at the present, we cannot recommend a definitive course of action. Breastfeeding is allowed, although the nipple should be carefully prepared, and breastfeeding should be discontinued if fissures appear or bleeding occurs. Pregnant women coinfected with HCV and HIV present a clearly greater risk of perinatal HCV transmission and therefore constitute an exception to these permissions.

\section{Prevention}

Unapparent HCV transmission in society and in the health care environment: What should we recommend to Health Oversight Agencies?

We recommend extremely rigorous inspection of health and esthetic institutes, as well as continuing education of the professionals who work in this area. We should also emphasize the need for ongoing education of health professionals at all levels of patient care - from basic care to highly complex treatments.

Vaccines: Which and when?

Susceptible individuals with hepatitis $C$ should be vaccinated against hepatitis $A$ and $B$. There is a real need to make vaccines against hepatitis A available in the public health care system.

\section{The Laboratory in the Era of Individualized Treatment}

Quantification of viral loads for all genotypes?

Yes, it is recommended that the viral load of all patients be quantified.

Which quantification method should be used?

It is recommended that the method used be reproducible, be sensitive, and present ample linearity.

'Moments of decision': At baseline (One measurement? When? Several measurements?), as well as at weeks 4, 12, and 24; end-of-treatment response (ETR), sustained virologic response_(SVR)...What else?

Baseline viral load should be determined prior to but as close as possible to the initiation of the treatment. It can be determined only once provided that the test is performed under ideal technical conditions. At week 4, the same test will be qualitative in order to define the presence of rapid virologic response (RVR), defined as the detection of no viral RNA. At week 12, it should be quantitative, adopting the criteria of no early virologic response (EVR) (a drop in viral load $\leq 2 \log 10$ ), partial EVR (drop $\geq 2 \log 10$ ), and complete EVR (no viral RNA detected). At week 24, the test will be qualitative. If viral RNA is detected, the treatment instituted exclusively for virologic purposes will have to be interrupted. Determination of the ETR - emphasizing the differences in duration in different genotypes and patients - as well as of the SVR, must be qualitative. The SVR should be determined at 24 weeks after the end of the treatment.

It should be noted that monitoring will be unnecessary during the treatment in the cases in which RVR occurred and adequate treatment compliance is maintained.

\section{'Week 12' SVR?}

Determination of the SVR at 12 weeks after the end of the treatment is not currently considered a useful or valid measure.

Metabolic alterations: Homeostasis model assessment (HOMA) and glucose tolerance test: when to order, how to interpret

Various studies indicate the role of hepatitis $\mathrm{C}$ as a factor implicated in the development of type 2 diabetes in patients at high risk (male gender, over 40 years of age, and overweight). In experimental models, HCV was found to induce insulin resistance, including increased production of tumor necrosis factor as one of the contributing factors. Insulin resistance is also associated with the development of steatosis and progression of liver fibrosis, principally in patients infected with HCV genotype 1. Therefore, there seems to be an association between insulin resistance and the characteristics associated with patients presenting a worse response to the hepatitis C treatment: cirrhosis, obesity, concomitant infection with HIV (taking antiretroviral drugs), etc.

In summary: HCV promotes insulin resistance, which leads to steatosis, fibrosis, and resistance to treatment with interferon alpha (IFN- $\alpha)$.

The HOMA mathematical model [(serum levels of fasting insulin vs. serum levels of fasting glucose)/22.5] has proven useful in the evaluation of sensitivity to insulin; however, it has not been completely standardized for all clinical situations, which is why its routine use is not recommended, except in the situations that will be reviewed now (metabolic alterations) and in the Proceedings.

\section{Noninvasive monitoring of fibrosis}

\section{Value of simplicity: Thrombocytopenia as a marker of liver cirrhosis}

Thrombocytopenia in an individual with hepatitis C indicates moderate to severe liver fibrosis. The sensitivity of this diagnostic marker of advanced fibrosis increases when it is used in combination with other biochemical tests, such as determination of the aspartate aminotransferase to platelet ratio index, calculated using the formula: aspartate aminotransferase/alanine aminotransferase ratio + platelets. 
When is it indicated?

Noninvasive monitoring of liver fibrosis should be performed in cases of (i) contraindication or difficult access to liver biopsy, (ii) the need for such monitoring in order to hasten the performance of a new biopsy in patients at greater risk of progression (immunocompromised patients) or in individuals presenting stage F1 fibrosis, and (iii) in patients with liver cirrhosis under treatment for modulation of fibrosis (IFN maintenance).

\section{How to measure: Serum panels vs. Fibroscan}

Sensitivity and positive predictive value are increased by combining the methods.

Liver biopsy is dead. . . Long live liver biopsy!

\section{Biopsy for all genotypes? And for all patients?}

Yes, except for clinical contraindications, the biopsy should be performed for all patients presenting detectable levels of HCV RNA.

The central issue is the absolute need for the biopsy sample to be representative of the hepatic parenchyma, since inappropriate biopsies frequently result in understaging of the disease. It is highly recommended that a needle biopsy be performed (wedge biopsies produce subcapsular samples that cannot be used in the staging of fibrosis nor in the staging of inflammation); needles that produce very thin biopsies highly limit architectural staging and should not be used. Trucut 14gauge needles or needles that produce samples of equivalent diameter, measuring at least $1.5 \mathrm{~cm}$ long and/or 10 represented portspaces, are recommended. If the biopsy is performed during a surgical procedure, it should be done at the outset of the procedure in order to avoid the artifacts that surgery can produce in the liver tissue.

The biopsy report should include a staging system and scoring (METAVIR or SBP, preferably). It should be noted, however, that the pattern of inflammatory alterations (portal, interface, and lobular components) should be described in detail, since it has become increasingly important in predicting the evolution of the disease as well as in the differential diagnosis with other diseases. In addition, the principal role of biopsy is to rule out other liver diseases - steatohepatitis (alcoholic or nonalcoholic), for example, frequently co-exists with hepatitis $\mathrm{C}$ and is known to have a significant impact on the evolution of the disease.

Value of the imaging and serum level methods in the diagnosis of liver fibrosis: who needs endoscopy?

Prior to biopsy, patients with indirect evidence of portal hypertension (ultrasonographic signs, thrombocytopenia) should be submitted to endoscopy of the upper digestive tract for detection of esophageal varicose veins that would render a liver biopsy unnecessary in the diagnosis of liver cirrhosis.

\section{Clinical Management in Borderline Situations}

Portal hypertension in clinical practice: Clinical management of pretreatment thrombocytopenia

There is no conclusive evidence on the management of pretreatment thrombocytopenia; however, some alternatives can be considered and are reviewed in the Proceedings.
Liver transplant in clinical practice: post-transplant limitations. Fibrogenesis, treatment after transplant, rejection control and live donor

Liver disease caused by HCV corresponds to almost half of the indications for liver transplant. Currently, in Brazil, the distribution of the organ follows a criterion of severity, using the model for end-stage liver disease (MELD). After the transplant, up to $80 \%$ of the patients experience histologic recurrence, and the pre-transplant viral load is one of the most significant risk factors. For this reason, pre-transplant treatment should always be considered, even in patients with decompensated cirrhosis, provided that the treatment is given in a specialized center and with an active transplant treatment team. Post-transplant treatment for chronic hepatitis is generally indicated when fibrosis is $\geq 2$ or when periportal activity is $\geq 3$. Although the duration of treatment should be at least 48 weeks, it should be individualized according to the virologic response profile.

\section{Treatment}

Nondrug and nonspecific HCV treatment: metabolic syndrome, nonalcoholic steatohepatitis, diabetes, obesity - evidence for the use of diet, hypolipidemic agents, and antidiabetic medication

It is recommended that the conditions associated with worsening of liver fibrosis and lower SVR rate, such as obesity, type 2 diabetes, nonalcoholic steatohepatitis, steatosis, dyslipidemia, and metabolic syndrome, be brought under control before antiviral treatment is instituted.

Patients with concomitant diseases; how to use IFN- $\underline{\alpha}$ and specific measures for drug users and patients with mental disease. Socially marginalized individuals and prisoners. Individuals with kidney disease in pre- and post-kidney transplant phases. Individuals with auto-immune disease and extrahepatic manifestations

At facilities where a multiprofessional approach is taken or at specialized centers, both of which allow appropriate monitoring and control of all clinical situations of the underlying disease, as well as of those potentiated or triggered by HCV treatment, it is recommended that HCV treatment be instituted first. It is noteworthy that, in the case of auto-immune manifestations (cryoglobulinemia and auto-immune hepatitis in particular), treatment for HCV should be given - observing the premises above. Corticosteroids and other immunosuppressants can be used concomitantly when indicated.

Ribavirin: Always a full dose? And how much (0.8 g, 1 g, 11 $\mathrm{mg} / \mathrm{kg} /$ day, $15 \mathrm{mg} / \mathrm{kg} /$ day)?

Ribavirin should always be given in a full dose. The recommended dose is $15 \mathrm{mg} / \mathrm{kg} /$ day or $1 \mathrm{~g}$ for patients who weigh $<75 \mathrm{~kg}$. The minimum dose to be considered in adjustments is $11 \mathrm{mg} / \mathrm{kg} / \mathrm{day}$.

Common adverse effects: how to deal with anemia, neutropenia and thrombocytopenia before the treatment, during the treatment, and triggered by the treatment? What are the basal limits for patients with and without cirrhosis to be treated? How can the effects be reversed? 
The minimum criteria for treatment should be hemoglobin $\geq 10 \mathrm{~g} / \mathrm{dL}$, neutrophils $>1500 / \mathrm{mm}^{3}$ and platelets $>60,000 / \mathrm{mm}^{3}$. Patients presenting a drop in hemoglobin to $<10 \mathrm{~g} / \mathrm{dL}$ or a drop $\geq 3.5 \mathrm{~g} / \mathrm{dL}$ with clinical manifestations should be given Erythropoietin in a dose of 40,000 IU/week until the end of the treatment or until anemia is under control. Patients in whom neutrophils drop to $<750 / \mathrm{mm}^{3}$ should receive filgrastim in a dose of $300 \mu \mathrm{g}$ one to three times a week until neutropenia is under control. In patients presenting a drop in platelets to < $25.000 / \mathrm{mm}^{3}$, the treatment should be discontinued. Adjustment regimens of the dose of IFN with intermediate values are presented in the Proceedings.

When to treat: the patient, the doctor, the system ...

The patient wants to be treated. How can we deal with this?

There is no definite position on this situation. We emphasize the need to give clear and exhaustive information regarding the indications for treatment and the factors associated with the progression of the disease.

Is early indication of treatment worthwhile? In which cases?

Treatment can be considered for individuals with level 1 structural lesion (F1 METAVIR), who present incomplete septa, A3 activity (METAVIR), who are under 60 years of age, and present co-factors associated with risk of progression (obesity, nonalcoholic steatohepatitis, etc.).

Use of IFN and ribavirin

The patient, the virus, and the medication: Relevant factors in predicting response before and during the treatment

There has been no relevant alteration regarding the elements described. Please see the concepts listed below regarding individualization based on EVR.

Is conventional treatment with IFN- $\alpha$ dead? If not, when should we use it?

It is the understanding of the Brazilian Society of Infectious Diseases that treatment with conventional IFN- $\alpha$ is no longer justifiable and should not be performed.

Dose of IFN, the 'burden' of the 'weight'

The dose of pegylated IFN- $\alpha-2$ a (PEG-IFN- $\alpha-2 a$ ) should be $270 \mu \mathrm{g} /$ week for patients weighing $>85 \mathrm{~kg}$ and presenting a high viral load ( $>850,000 \mathrm{IU} / \mathrm{mL})$. The dose of PEG-IFN- $\alpha-2 b$ should be $1.5 \mu \mathrm{g} / \mathrm{kg} /$ week.

\section{Short, long, standard treatment ... Always individualize?}

In patients presenting a RVR, no comorbidities (severe steatosis or cirrhosis), and a low viral load $(<250,000$ IU/ $\mathrm{mL}$ for genotype 1 ), the duration of the treatment can be reduced: to 12 weeks for genotypes 2 and 3; and to 24 weeks for genotype 1 . Patients with a high viral load and complete EVR should be treated for the standard duration, and patients presenting a slow response (no RVR, partial EVR and negative at week 24) should be treated for an additional 24 weeks.
The fear of suspending treatment: How should we deal with that decision?

We emphasize that, if the objective of the treatment is virologic, the absence of EVR and viral detection at week 24 necessarily imply the interruption of the treatment.

Maintenance treatment with IFN: When and how? Is 'watchful waiting' still a valid concept?

Maintenance treatment with PEG-IFN- $\alpha$ is considered for the following patients: those with structural lesion > F3 and/ or signs of portal hypertension (esophageal varicose veins, splenomegaly, dilated blood vessels, etc.) and/or platelet counts $<110,000 / \mathrm{mm}^{3}$ who are categorized as Child-Pugh class A or B, with no history of severe or potentially uncontrollable decompensation; those without hepatocellular carcinoma; relapsers; and partial responders or nonresponders to the combination of PEG-IFN- $\alpha$ and ribavirin (or to IFN only when ribavirin is contraindicated) administered with adequated compliance and for a minimum of 12 weeks. Maintenance treatment with PEG-IFN- $\alpha$ should also be considered for patients for whom the full dose treatment is contraindicated. Child-Pugh class $\mathrm{C}$ patients on the transplant waiting list should be treated in specialized centers. Proposed regimen²: PEG-IFN- $\alpha$-2b, 0.5-1.0 $\mu \mathrm{g} / \mathrm{kg} /$ week subcutaneously (sc); PEGIFN- $\alpha-2 a, 90 \mu \mathrm{g} /$ week (sc). Duration ${ }^{2}$ : Minimum of 24 months, indefinite, or even until there is an antiviral treatment that is proven to be safe and efficient. Monitoring the occurrence of complications (hepatocellular carcinoma, gastrointestinal bleeding, encephalopathy, etc.)

Monotherapy with ribavirin, although previously described, cannot be recommended at the moment, due to insufficient evidence.

Impact of reduction in the dose of IFN and/or ribavirin at various moments of the treatment

Every effort should be made to always maintain full treatment.

Positive and negative predictive values in clinical practice: patients mono- and co-infected with HIV

Positive predictivity data in the presence of RVR and negative predictivity data in the absence of EVR are equally valid for HCV mono-infected patients and patients co-infected with HCV and HIV.

\section{Retreatment: When and how?}

Retreatment will always be considered when the previous treatment was considered suboptimal for the current concepts (poor compliance, insufficient doses, inappropriate interruptions, inadequate management of adverse events, uncontrolled comorbidities, etc.) and when there is recurrence. The treatment in true nonresponders to PEG-IFN and ribavirin presents such a low chance of success that it cannot be routinely recommended.

Due to the fact that relapsers/nonresponders to initial treatment constitute a quite heterogeneous group, it is

${ }^{2}$ Dose schedule and duration were suggested based on preliminary data and should be re-evaluated taking into account the results of ongoing studies. 
necessary to qualify and carefully select the patients that should be retreated. Various factors that might have influenced the nonresponse should be modified before the initiation of or even during the new therapeutic cycle. Currently, patients considered less likely to respond to retreatment are those who are true nonresponders, those who are of the Black race, those infected with genotype 1, those with high viral loads, those with advanced liver disease, and those who present intercurrent conditions (obesity, etc.)

Patients previously treated with IFN as monotherapy or with the combination of IFN and ribavirin stand a greater chance of presenting an SVR than do nonresponders to the combination of PEG-IFN and ribavirin. Those who experienced recurrence during the treatment (breakthrough) or after the treatment fare better than do true nonresponders. Patients who are noncompliant with previous treatment, as well as those who required reduced doses of IFN or ribavirin due to cytopenia or other adverse effects, usually respond better to retreatment than do those who received full doses. Of course, the factors responsible for noncompliance should be eliminated, and reductions in medication doses should be properly approached. In nonresponders, it is recommended that erythropoietin and filgrastim be started sooner, and further dose reductions should be avoided at all costs. Users of drugs or alcohol who, due to their addiction, did not adequately comply with all the phases of the previous treatment, can more adequately respond to retreatment provided that these cofactors are nullified. This also applies to patients who did not receive adequate social or cultural support. Patients who suffer from obesity, insulin resistance, dyslipidemia, steatosis, anemia, or liver diseases (e.g., hemochromatosis) should be retreated, preferably after the proper diagnosis and treatment of these concomitant conditions. Doses of PEG-IFN should be the same as those used for treatmentnaïve patients. We believe that, in retreatment regimens, the doses of ribavirin should be as high as possible. Due to the paucity of studies with large patient samples, we recommend that the duration of retreatment be 48 weeks for all genotypes. At the moment, there are no consolidated data in the literature to support using higher doses of PEGIFN or ribavirin, using induction doses, or extending the treatment time to more than 48 weeks in retreatment cases. Regarding the week 12 rule, there is strong evidence that patients who do not present negativity for HCV RNA by this time will have very little chance of presenting an SVR, and their treatment should be interrupted.

\section{IFN: Backbone of the current treatment. And the future? Perspectives and frustrations}

Preliminary results of developing therapies show that IFN$\alpha$ will still be the active principle of the treatment for many years. For this reason, we have adopted several of the practices that are presented here.

\section{Co-infections}

Antiretroviral therapy 2007 and HCV treatment: relevant interactions

Although ribavirin can reduce the concentration of some antiretroviral nucleoside analog reverse transcriptase inhibitors (NARTIs), there seem to be no clinical consequences.

The adverse effects of NARTIs have been associated with HCV co-infection, female gender, obesity and prolonged exposure to these drugs. The principal combinations with less hepatic repercussion among NARTIs are as follows: lamivudine + abacavir; lamivudine + zidovudine; lamivudine + abacavir + zidovudine, and emtricitabine + tenofovir. However, current studies indicate that ribavirin interferes with the effect of abacavir. Therefore, ribavirin should be used with caution in patients receiving the highly active antiretroviral therapy regimen and being treated for HCV.

Greater toxicity is principally caused by concomitant use of didanosine and ribavirin.

CD4 counts: Minimum limits for treatment and decisionmaking in patients with reconstituted immunity or immunocompetent patients

Precise indication of therapy in HIV/HCV co-infected patients can be made in patients with CD4 counts $>350$ cells/ $\mathrm{mm}^{3}$. In patients with CD4 counts between 200 and 350 cells/ $\mathrm{mm}^{3}$, the decision to treat HCV infection should take into account other factors, such as the duration of HCV infection, the severity of the liver disease, the level of HIV suppression and classical predictors of treatment response to HCV, such as genotype and viral load.

The SVR can be predicted when HCV RNA is undetectable in serum by week 4 of treatment. However, a $<2 \log 10 \mathrm{IU} / \mathrm{mL}$ reduction in viral load by week 12 and/or viremia detected at week 24 predict a lack of virologic response, and discontinuation of the treatment is indicated.

Ongoing studies are evaluating 72-week maintenance therapy in co-infected patients and week-24 nonresponders. This might be the truly appropriate treatment duration for HIV-positive patients, even with longer time and smaller fractionated doses.

Is the immunocompetent HIV/HCV co-infected patient 'monoinfected'?

Yes, the evolution is quite similar.

Multiple viral infections: Who should we treat first and how?

Care should be given to the dominant virus between HBV and HCV. When treating the dominant virus, the other might become active. We need to quantify the HBV DNA in order to manage this situation.

The HTLV apparently modulates the host immune response, a fact that should be considered and which is detailed in the Proceedings.

There is no consensus regarding the correct course of action in multiple co-infections. 